\title{
B and Upsilon Cross Sections at HERA-B
}

\author{
Antonio Sbrizzi * \\ University of Bologna - Physics Department \\ via Irnerio 46, I-40126 Bologna - Italy
}

\begin{abstract}
A new measurement of the $b \bar{b}$ and $\Upsilon$ production cross sections using HERA-B data is presented [1]. During the 2002/2003 run, HERA-B recorded approximately 150 million $p A$ dilepton trigger events in which $150,000 \mathrm{~J} / \psi \rightarrow e^{+} e^{-}$and $100,000 \mathrm{~J} / \psi \rightarrow \mu^{+} \mu^{-}$ decays have been reconstructed. The $b$ events are tagged via inclusive bottom quark decays into a $\mathrm{J} / \psi$, by exploiting the longitudinal separation of $\mathrm{J} / \psi \rightarrow l^{+} l^{-}$decay vertices from the primary $p A$ interaction. In the dimuon channel, $b$ events are also tagged via double semi-leptonic $b$ decays. The $\Upsilon$ cross section is measured in the dimuon and dielectron decay channels.
\end{abstract}

\section{Introduction}

The measurement of bottom production in fixed target collisions offers the possibility to test perturbative QCD in the near threshold energy regime, where the effect of higher order processes, such as soft gluon emission, has been calculated $[2,3]$. These calculations have large uncertainties due to the $b$ quark mass and the dependence on the normalization scale.

The published experimental results are inconsistent, even though they were obtained in similar experimental conditions by searching for $\mathrm{J} / \psi[4]$ and semi-leptonic [5] decays of $b$ hadrons. HERA-B recently published the most accurate result based on independent measurements of $\mathrm{J} / \psi[6]$ and double muonic $b$ decays [7].

For $\Upsilon$ production, several measurements are available in the HERA-B energy region $(\sqrt{s}=41.6 \mathrm{GeV})$, but the results disagree.

\section{HERA-B detector and data sample}

HERA-B is a forward spectrometer installed at the $920 \mathrm{GeV}$ proton storage ring of DESY. The acceptance is $[15,220] \mathrm{mrad}$ in the bending plane and $[15,160] \mathrm{mrad}$ in the vertical plane), which approximately corresponds to a Feynman- $x\left(x_{\mathrm{F}}\right)$ of $[-0.35,0.15]$. Charged particle tracks produced in the interactions of the proton beam halo with wires of different materials $\left({ }^{12} \mathrm{C},{ }^{48} \mathrm{Ti}\right.$ and $\left.{ }^{184} \mathrm{~W}\right)$ are tracked with a silicon microstrip detector [8] whose first station (of 8 ) is a few centimeters from the target system and which extends approximately $2 \mathrm{~m}$ further downstream. A primary vertex resolution of $500 \mu \mathrm{m}$ along the beam and $50 \mu \mathrm{m}$ in the perpendicular plane is achieved. Up to $13 \mathrm{~m}$ downstream of the target, honeycomb chambers in the outer region $[9,10]$ and microstrip gaseous chambers in the inner region [11] allow to track particles and to measure their momenta from the bending in a $2.13 \mathrm{~T} \cdot \mathrm{m}$ vertical magnetic field. A Cherenkov detector [12] is used for $\pi / K / p$ separation. An electromagnetic shashlik calorimeter [13] serves for $e$ and $\gamma$ identification. At the rear of the detector, muons with momenta larger than $5 \mathrm{GeV} / c$ are tracked with triple stereo layers of gaseous tube chambers interleaved with hadron absorbers [14].

In the 2002/2003 run, a multilevel dilepton trigger [15] allowed to record 150 million events of $p A$ interactions with about 250,000 reconstructed $\mathrm{J} / \psi \rightarrow l^{+} l^{-}$decays (Fig. 1).

${ }^{*}$ On behalf of the HERA-B collaboration. 

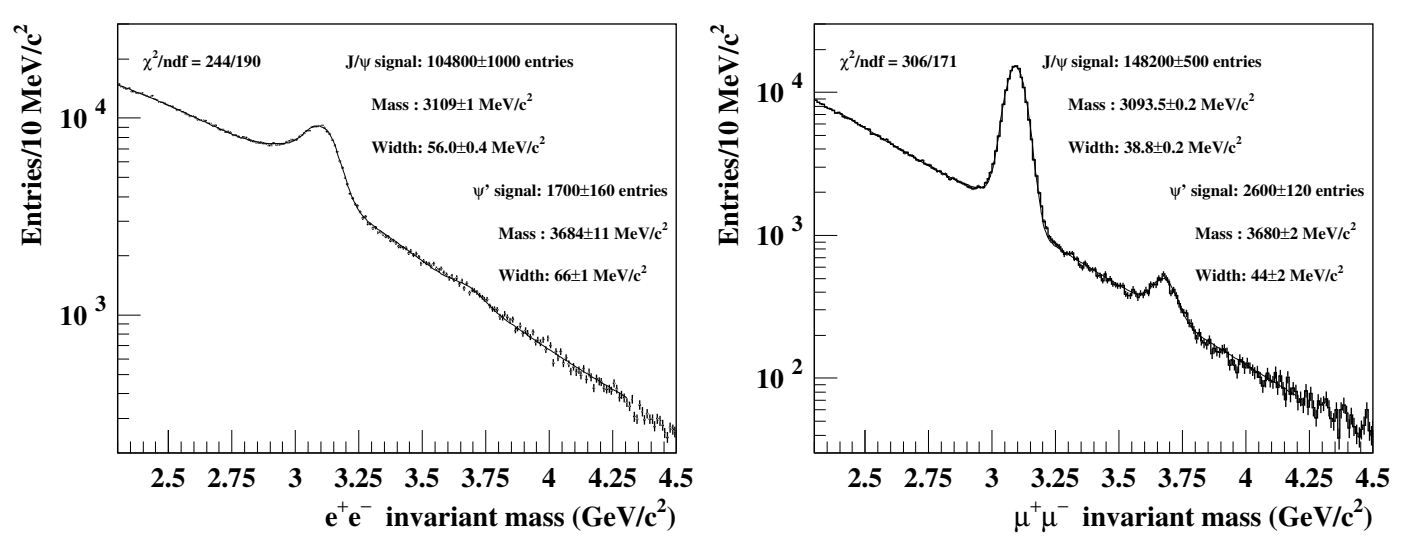

Figure 1: Invariant mass of unlike-sign dielectrons (left) and dimuons (right).

The systematic uncertainties due to the detector performance and acceptance is reduced by normalising the $b \bar{b}$ and $\Upsilon$ production cross sections to the prompt $\mathrm{J} / \psi$ cross section, as obtained from a NRQCD fit to world data $\left(\sigma_{\mathrm{J} / \psi}=502 \pm 44 \mathrm{nb} /\right.$ nucleon [16]).

\section{B production}

Bottom quarks are produced in $b \bar{b}$ pairs at HERA-B. The $b \bar{b}$ cross section is extracted from data with two statistically independent measurement methods. In a first method, $b \bar{b}$ events are identified with the inclusive $\mathrm{J} / \psi$ decay of a $b$ or $\bar{b}$ hadron. In a second method, $b \bar{b}$ events are tagged by searching for simultaneous semi-leptonic decays of the $b$ and the $\bar{b}$ hadron.

\subsection{Detached $\mathrm{J} / \psi$ analysis}

In order to distinguish a prompt $\mathrm{J} / \psi$ from those coming from a $b$ hadron decay, the $b$ hadron lifetime is exploited. The typical path length of $b$ hadrons at HERA-B is about $9 \mathrm{~mm}$, well above the experimental resolution of the longitudinal primary vertex position $(0.5 \mathrm{~mm})$. Since there are no other long-lived particles decaying into a $\mathrm{J} / \psi$, a $\mathrm{J} / \psi$ vertex which is detached from the primary vertex is a unique indication of a $b$ hadron decay event.

The $b \bar{b}$ cross section can be expressed as

$$
\sigma_{b \bar{b}}^{A}=\frac{n_{b \bar{b}}}{n_{\mathrm{J} / \psi}} \cdot \frac{\sigma_{\mathrm{J} / \psi}^{A}}{\epsilon_{R} \cdot \epsilon_{b \bar{b}}^{\Delta} z \cdot \mathrm{BR}(b \rightarrow \mathrm{J} / \psi+X)},
$$

where $\sigma_{\mathrm{J} / \psi}$ is the prompt $\mathrm{J} / \psi$ cross section, $n_{b \bar{b}}$ and $n_{\mathrm{J} / \psi}$ are the measured numbers of $b$ and prompt $\mathrm{J} / \psi$ decay events; $\epsilon_{R}$ is the ratio of the $\mathrm{J} / \psi$ selection efficiency for $b$ and prompt $\mathrm{J} / \psi$ events; $\epsilon_{b \bar{b}}^{\Delta} z$ is the efficiency of the detachment cuts. The dependency of the production cross sections on the mass number $A$ is parameterized as $\sigma^{A}=\sigma_{0} \cdot A^{\alpha}$, where $\sigma_{0}$ stands for the proton-nucleon cross section, and the parameter $\alpha$ stands for any possible nuclear effect. For $b \bar{b}$ production no nuclear effect is expected $(\alpha=1)$. For $\mathrm{J} / \psi$ production, the value measured by the E866 experiment ( $\alpha=0.96 \pm 0.01$ [17]) is used. The branching 
ratio $\mathrm{BR}(b \rightarrow \mathrm{J} / \psi+X)$ was measured at LEP1 from $Z$ decays $(0.0232 \pm 0.0020$ [18]). The efficiencies are determined through MC simulations.

A lepton track must have segments in the vertex detector and tracking systems, in addition to a set of particle identification requirements. When two opposite charge leptons are found, a vertex fit is performed. Figure 1 shows the dilepton mass distributions of reconstructed vertices, where the prompt $\mathrm{J} / \psi$ signal is visible.

In order to identify a detached lepton pair coming from a $b$ decay, the longitudinal separation between the $\mathrm{J} / \psi$ vertex and the wire target, the lepton and $\mathrm{J} / \psi$ impact to the wire are exploited. The final selection procedure is obtained by a blind optimisation aiming at maximising the significance of the MC signal over the background estimated from both $\mathrm{MC}$ and data. Figure 2 shows the invariant mass distributions, upstream and downstream of the target, when the optimised selection criteria are applied.
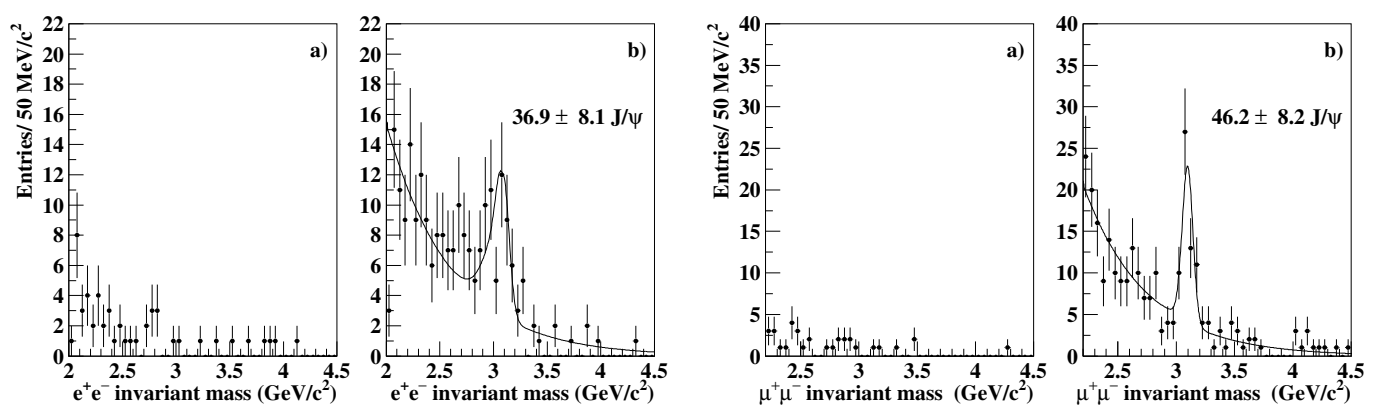

Figure 2: Dielectron (left) and dimuon (right) invariant mass distributions after vertex detachment cuts. The plots show the target upstream region (a), which is unphysical (combinatorial background) and the target downstream region (b) where $b \rightarrow \mathrm{J} / \psi+X$ decay events are visible. The solid line shows the result of a likelihood fit.

The downstream spectra in the $\mathrm{J} / \psi$ mass region are dominated by $b$ decays events. Background consists of combinatorial lepton pairs (44\%) and simultaneous semi-leptonic decays of $b \bar{b}(43 \%)$ and $c \bar{c}(13 \%)$ pairs. Combinatorial background is estimated from the unphysical upstream events, while bottom and charm background are obtained from MC simulations.

The measured number of $b$ decays events results from an unbinned likelihood fit of the mass spectra. The result for the combined muon and electron channels is $n_{b \bar{b}}=83 \pm 12$ and, combined with a previous HERA-B measurement [19], it corresponds to a production cross section $\sigma_{b \bar{b}}=14.9 \pm 2.2_{\text {stat }} \pm 2.4_{\text {sys }} \mathrm{nb} /$ nucleon in the full $x_{\mathrm{F}}$ range.

A confirmation of the $b$-flavour of the tagged $b$ events is given by the measured lifetime $(1.39 \pm 0.19 \mathrm{ps}[6])$ which is compatible with the expectation (1.54 ps [18]).

\subsection{Double semi-leptonic $b$ decays}

After production, $b \bar{b}$ pairs hadronise and mostly decay into $c$ hadrons. Since $b$ and $c$ hadrons have a large probability to decay with the emission of a muon (semi-muonic decay) [18], the $b \bar{b}$ production cross section is measured by searching for $b \bar{b} \rightarrow \mu^{+} \mu^{-}+X$ decay events, 
in which at least two of the four heavy quarks typically produced in a $b \bar{b}$ event $(b, \bar{b}, c, \bar{c})$ undergo semi-muonic decays (double muonic b decays).

The $b \bar{b}$ cross section can be expressed as [20]

$$
\sigma_{b \bar{b}}^{A}=\frac{n_{b \bar{b}}}{n_{\mathrm{J} / \psi}} \cdot \frac{\sigma_{\mathrm{J} / \psi}^{A} \cdot \mathrm{BR}\left(\mathrm{J} / \psi \rightarrow \mu^{+} \mu^{-}\right) \cdot \epsilon_{\mathrm{J} / \psi}}{\sum_{j} \mathrm{BR}_{j}\left(b \bar{b} \rightarrow \mu^{+} \mu^{-}+X\right) \cdot\left(1-\theta_{j}\right) \cdot \epsilon_{b \bar{b}, j}},
$$

where $\epsilon_{\mathrm{J} / \psi}$ is the prompt $\mathrm{J} / \psi$ selection efficiency and $\mathrm{BR}(\mathrm{J} / \psi)=5.93 \pm 0.06 \%$ [18]. All possible decays originating from a $b \bar{b}$ pair and leading to a dimuon final state, having branching $\mathrm{BR}_{j}\left(b \bar{b} \rightarrow \mu^{+} \mu^{-}+X\right)$ and efficiency $\epsilon_{b \bar{b}, j}$, are included. The factor $\theta_{j}$ accounts for the effect of neutral $B$ meson mixing.

The $b \bar{b}$ event selection is based on a pair of oppositely charged muons not coming from the primary interaction vertex, having a large momentum transverse to the beam. The search of double semileptonic events is only performed in the muon channel due to the larger systematic uncertainty associated with the electron identification. The $\mathrm{J} / \psi$ mass region is excluded to be statistically independent from the result provided by the detached $\mathrm{J} / \psi$ analysis.

The measured number of double muonic $b$ decay events $\left(n_{b \bar{b}}\right)$ is obtained with a multiparameter likelihood fit to the data of the simulated transverse momentum $\left(p_{\mathrm{T}}\right)$ and impact parameter $(I p)$ distributions of signal and background events (Figure 3 ).

The background consists of double muonic decays of $c$ hadrons and random combinations of muons from decay of low mass mesons (combinatorial background). Muons from Drell-Yan events are negligible. The result of the likelihood fit is $n_{b \bar{b}}=$ $83 \pm 12$, which corresponds to a production cross section of $\sigma_{b \bar{b}}=17.5 \pm 2.6_{\text {stat }} \pm$ $3.3_{\text {sys }} \mathrm{nb} /$ nucleon.

The combined result of the HERA-B measurements based on detached $\mathrm{J} / \psi$ and double semileptonic $b$ decays is $\sigma_{b \bar{b}}=15.8 \pm$ $1.7_{\text {stat }} \pm 1.3_{\text {sys }}^{\text {uncorr. }} \pm 2.0_{\text {sys }}^{\text {corr. }} \mathrm{nb} /$ nucleon.
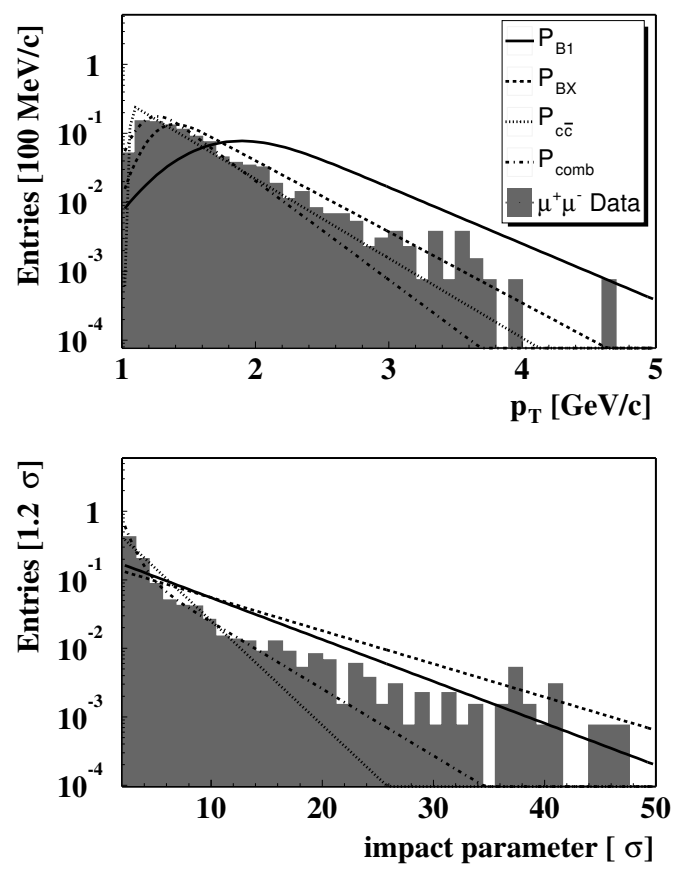

Figure 3: Simulated $p_{\mathrm{T}}$ (top) and Ip (bottom) distributions of double muonic $b$ and $c$ decays, combinatorial background and oppositely charged dimuon data.

\section{$4 \Upsilon$ production}

Dilepton decays are used to study $\Upsilon$ production. The selection criteria are similar to those applied for $\mathrm{J} / \psi$ identification. 
Due to the broad signal and the low number of signal events over background, it is important to have a good description of the background. Combinatorial events (estimated by like-sign pairs from real data) and the Drell-Yan process (estimated from MC simulation) are the main sources of background. These two backgrounds are shown in Fig. 4, together with the $\Upsilon$ signals in the muon and electron channel.
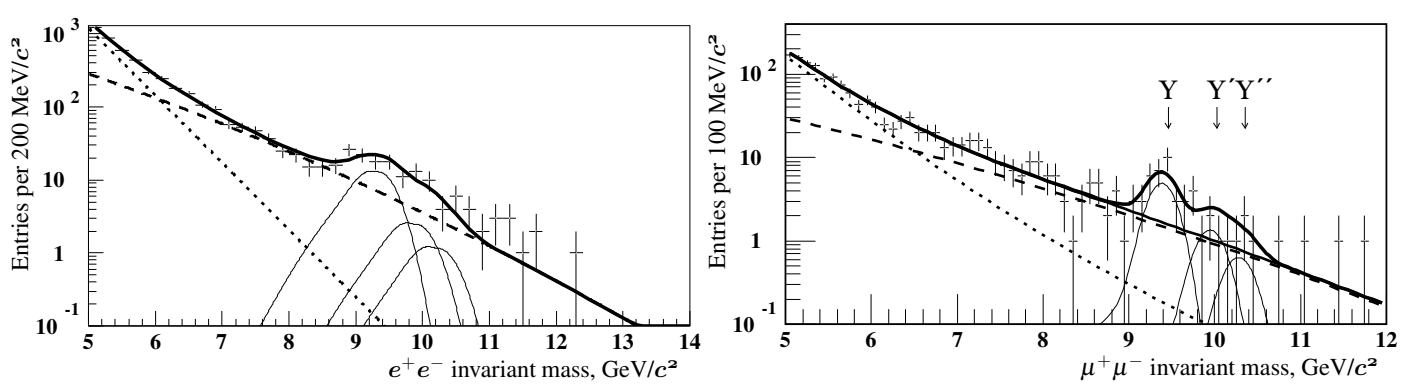

Figure 4: Dielectron (left) and dimuon (right) invariant mass spectra. Solid lines represent combinatorial and Drell-Yan background.

A good and stable fit of the signal is obtained by fixing the background shapes and the relative production ratios of $\Upsilon(1 S) / \Upsilon(2 S) / \Upsilon(3 S)$ to the E605 results [21]. The differential cross section at central rapidity times the branching ratio can be express as

$$
\left.\operatorname{BR}\left(\Upsilon \rightarrow l^{+} l^{-}\right) \frac{d \sigma_{\Upsilon}}{d y}\right|_{y=0}=\operatorname{BR}\left(\mathrm{J} / \psi \rightarrow l^{+} l^{-}\right) \cdot \sigma_{\mathrm{J} / \psi} \cdot \frac{n_{\Upsilon}}{n_{\mathrm{J} / \psi}} \cdot \frac{\epsilon_{\mathrm{J} / \psi}}{\epsilon_{\Upsilon}} \cdot \frac{1}{\Delta y_{\text {eff }}}
$$

where $\sigma_{\mathrm{J} / \psi}$ is the $\mathrm{J} / \psi$ production cross section, $n_{\Upsilon}$ and $n_{\mathrm{J} / \psi}$ are the numbers of observed $\Upsilon$ and $\mathrm{J} / \psi$ decays, respectively, and $\epsilon_{\mathrm{J} / \psi}$ and $\epsilon_{\Upsilon}$ are the $\mathrm{J} / \psi$ and $\Upsilon$ trigger and reconstruction efficiencies. The $\Upsilon$ production model gives $\Delta y_{\text {eff }}=1.068 \pm 0.002$ at $\sqrt{s}=41.6 \mathrm{GeV}$. The $\mathrm{J} / \psi$ cross section and branching ratios are the same as those used in the open $b \bar{b}$ analysis.

Combining muon and electron channels, a central rapidity production cross section of $\operatorname{BR}\left(\Upsilon \rightarrow l^{+} l^{-}\right) \frac{d \sigma_{\Upsilon}}{d y}=4.5 \pm 1.1 \mathrm{pb} /$ nucleon is obtained, a value in between the E605 and E772/E771 measurements [22, 23]. The spectrum of the world available experimental data is fitted with Craigie's parameterization [24] (see Fig. 5). An additional parameter $\alpha$ is used for nuclear suppression and yields a result compatible with no nuclear suppression.

\section{Conclusions}

A $b \bar{b}$ cross section of $\sigma_{b \bar{b}}=15.8 \pm 1.7_{\text {stat }} \pm 1.3_{\text {sys }}^{\text {uncorr. }} \pm 2.0_{\text {sys }}^{\text {corr. }}$ nb/nucleon is extracted from the largest sample of $b$ events recorded in fixed target experiments (176 events). The result is consistent with the latest QCD predictions of Bonciani et al. [2] and Kidonakis et al. [3] (see Fig. 5). The $\Upsilon$ differential cross section measurement at central rapidity times the branching ratio yields a value of $4.5 \pm 1.1 \mathrm{pb} /$ nucleon. A global fit to the $\Upsilon$ data shows that the result is consistent with no nuclear suppression (see Fig. 5). 

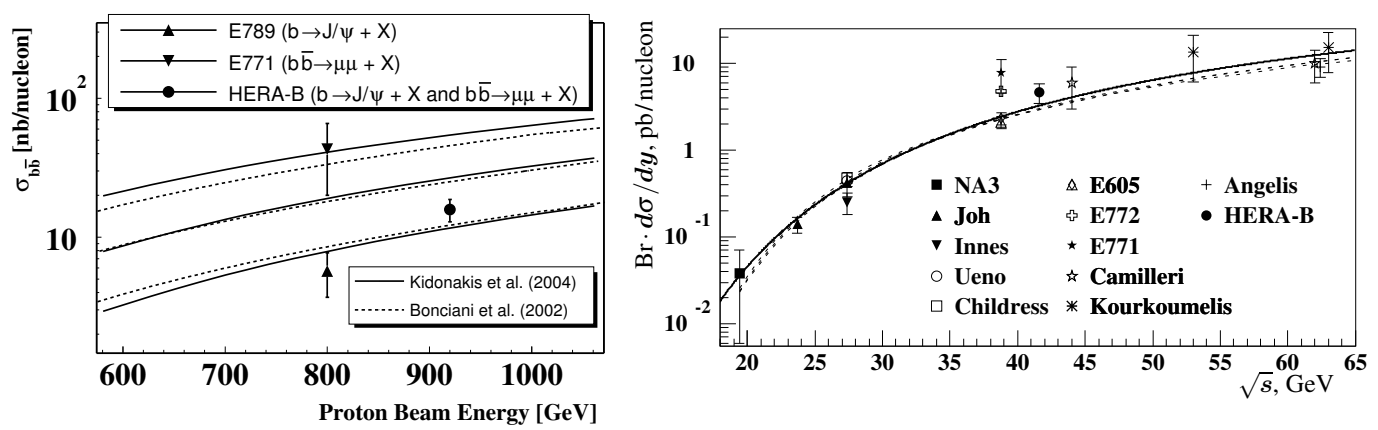

Figure 5: The left plot shows several measurements of the $b \bar{b}$ production cross section as a function of the proton energy in fixed target collisions. The theoretical predictions are superimposed. The right plot shows the $\Upsilon$ production cross section measured by different experiments, including HERA-B, and the modified Craigie parameterization fit.

\section{References}

[1] Slides: http://indico.cern.ch/contributiondisplay. py?contribid=237\&sessionid=5\&confid=9499.

[2] R. Bonciani et al.. Nucl. Phys., B529:424, 1998.

[3] N. Kidonakis and R. Vogt. Eur. Phys. J., C36:201, 2004.

[4] D. M. Jansen et al.. Phys. Rev. Lett., 74:3118, 1995.

[5] T. Alexopoulos et al.. Phys. Rev. Lett., 82:41, 1999.

[6] I. Abt et al.. Phys. Rev., D73:052005, 2006.

[7] I. Abt et al.. Phys. Lett., B650:103, 2007.

[8] C. Bauer et al.. Nucl. Instrum. Meth., A501:39, 2003.

[9] H. Albrecht et al.. Nucl. Instrum. Meth., A541:610, 2005.

[10] H. Albrecht et al.. Nucl. Instrum. Meth., A555:310, 2005.

[11] Y. Bagaturia et al.. Nucl. Instrum. Meth., A490:223, 2002.

[12] I. Ariño et al.. Nucl. Instrum. Meth., A516:445, 2004.

[13] G. Avoni et al.. Nucl. Instrum. Meth., A461:332, 2001.

[14] A. Arefiev et al.. IEEE Trans. Nucl. Sci., 48:1059, 2001.

[15] V. Balagura et al.. Nucl. Instrum. Meth., A494:526, 2002.

[16] F. Maltoni et al.. Phys. Lett., B638:202, 2006.

[17] M. J. Leitch et al.. Phys. Rev. Lett., 84:3256, 2000.

[18] W. M. Yao et al.. J. Phys., G33:1, 2006.

[19] I. Abt et al.. Eur. Phys. J., C26:345, 2003.

[20] A. Sbrizzi. PhD thesis, NIKHEF, Amsterdam, 2006.

[21] T. Yoshida et al.. Phys. Rev., D39:3516, 1989.

[22] T. Alexopoulos et al.. Phys. Lett., B374:271, 1996.

[23] P. L. McGaughey et al.. Phys. Rev., D50:3038, 1994.

[24] N. S. Craigie. Phys. Rept., 47:1, 1978. 